\title{
Bundesrat delegiert Rationierung an Mediziner
}

\author{
Bruno Henggi \\ Verantwortlicher Public Affairs der FMH
}

\author{
Die Ärzteschaft steht hinter drei von vier Tarifmassnahmen, sofern es gelingen \\ wird, im parlamentarischen Verfahren an der Tarif- und Organisationsautonomie \\ festzuhalten. Eine Massnahme lehnt die FMH dagegen kategorisch ab und wird sie \\ bekämpfen. Es handelt sich um den Versuch, Entscheide über Leistungsbeschrän- \\ kungen an die Ärztinnen und Ärzte zu delegieren.
}

Es war einmal durch das Departement des Innern (EDI) geplant gewesen, sämtliche 38 Massnahmen des Berichts der Expertengruppe zur Dämpfung des Kostenwachstums im Gesundheitswesen (Expertenbericht Verena Diener, 2017) in fünf Tranchen dem Parlament zur gesetzgeberischen Verarbeitung vorzulegen. Diese Pläne wurden im Bundesrat durchkreuzt. Auf der Agenda sind zwei Kostendämpfungspakete verblieben. Das zweite Paket wird Anfang nächsten Jahres in die Vernehmlassung geschickt und wird möglicherweise das Globalbudget enthalten, welches offiziell «verbindliche Zielvorgabe für das OKP-Wachstum» genannt wird. Das erste Kostendämpfungspaket hat der Bundesrat am 21. August 2019 verabschiedet und die Botschaft dem Parlament zugeleitet. Es enthält neun Massnahmen, wovon vier die Tarifgestaltung betreffen. Teil des Pakets sind ausserdem der Experimentierartikel, das Referenzpreissystem bei Arzneimitteln, die Rechnungskopie für Versicherte, das Beschwerderecht der Versicherer betreffend Beschlüsse der Kantonsregierungen zur Spitalplanung und Spitallisten. Neu enthält es als Massnahme separat ausgewiesen auch die Nennung der maximalen Bussenhöhe von CHF 20000 auf Gesetzesstufe, die gegen Leistungserbringer angeordnet werden kann, welche gegen vertragliche Abmachungen betreffend Wirtschaftlichkeit und Qualität der Leistungen (KVG Art. 56 und 58) sowie gegen Bestimmungen über die Rechnungsstellung (KVG Art. 42) verstossen.

\section{Massnahmen im Bereich der Tarifgestaltung}

Für die Einsetzung einer nationalen Tariforganisation (M34) für ambulante Behandlungen hat die FMH Unter- stützung signalisiert, sofern die Struktur- und Organisationsautonomie der Tarifpartner gewährleistet bleibt. Die FMH wird sich deshalb dafür einsetzen, die Vorlage des Bundesrats zu korrigieren. Für die gemeinsame Organisation im stationären Bereich (SwissDRG) gibt der Bundesrat zu Recht keine Grundsätze betreffend Form und Betrieb und schon gar nicht betreffend Finanzierung der Organisation vor. Für die gemeinsame Organisation der Tarifpartner, welche neu für ambulante ärztliche Behandlungen und nicht mehr für ambulante Behandlungen schlechthin zuständig sein soll, ist deshalb ebenfalls auf eine Bundeskompetenz zu verzichten.

Korrekturbedarf besteht auch im Bereich der Massnahme Pauschalen im ambulanten Bereich fördern (M15). Die FMH geht mit dem Bundesrat einig, dass Patientenpauschalen im ambulanten Bereich gefördert werden sollen. Das Ziel, die Anwendung solcher Pauschalen zu verbreitern, dürfte jedoch eher erreicht werden, wenn die Tarifstrukturen im Konsens und nicht gegen den Willen der Tarifpartner festgelegt werden. Es ist die nationale Tariforganisation, für welche Tarif- und Organisationsautonomie zu etablieren sind, die entscheiden soll, in welchen Bereichen der ambulanten Behandlung für Patientenpauschalen einheitliche Tarifstrukturen gelten sollen. Auf Ersatzvornahmen des Bundesrates ist zu verzichten. Dafür aber sollen einzelne Versicherer und Leistungserbringer dort, wo die Organisation (nach Art. 47a) keine Tarifstrukturen festgelegt hat, von sich aus Patientenpauschalen mit einer von ihnen gewählten Tarifstruktur vereinbaren können. Eine solche Disposition ist geeignet, zur Ausbreitung und Förderung von Patientenpauschalen beizutragen. 


\section{Tarif- und Organisationsautonomie ist zu gewährleisten}

Auch die Massnahme Tarifstruktur aktuell halten (M25) untergräbt in der Fassung des Bundesrats die Tarifautonomie. Sie kann nur dann unterstützt werden, wenn die geforderten Datenlieferungen in KVG Art. 47b Abs. 1 an die nationale Tariforganisation gehen und nicht an Bundesrat und Kantone. Der Bundesrat soll die Daten weiterhin erst dann erhalten, wenn die Tarifanpassung durch die Organisation erfolgt ist. Die Subsidiarität, welche ein Eingreifen des Bundesrats erst bei einem Scheitern der Tarifverhandlungen vorsieht, würde sonst zu Gunsten eines Staatstarifs aufgegeben.

Auf grundsätzliche Ablehnung stösst bei der FMH dagegen die Massnahme der Tarifpartner zur Steuerung der Kosten, welche in KVG Art. 47c der bundesrätlichen Reformvorlage abgebildet ist. Diese Massnahme war nicht Bestandteil des Expertenberichts Diener, sondern wurde vom EDI mit Hinweis auf die inhaltliche Verwandtschaft mit der parlamentarischen Initiative 17.402 Steuerung der Kosten im KVG durch die Vertragspartner ins erste Paket aufgenommen. In der Debatte im Nationalrat war ausgeführt worden, dass die Pa.Iv. 17.402 «im Wesentlichen Massnahme 1 des Expertenberichtes» entspreche. Und weiter wurde festgehalten: «Diese sieht die Festlegung von Globalzielen für die Kostenentwicklung vor» [1]. Nach materieller Diskussion wurde diese parlamentarische Initiative vom Nationalrat am 28. Mai 2018 mit 97:91 Stimmen abgelehnt. Das EDI nimmt in KVG Art. 47c nun einen zweiten Anlauf. Es will die Leistungserbringer und Versicherer verpflichten, in ihren Verträgen Massnahmen zur Steuerung der Kosten vorzusehen, und will den Bundesrat ermächtigen, diese Aufgabe subsidiär zu erfüllen.

\section{Ungenügende Tarifierung verstösst gegen das Versicherungsprinzip}

Die Botschaft kommentiert die Massnahme der Tarifpartner zur Steuerung der Kosten nicht frei von Ungereimtheiten. Grundsätzlich bestehe ein inhaltlicher Zusammenhang mit der Massnahme 1 aus dem Expertenbericht (Zielvorgabe für das OKP-Kostenwachstum), wird auf Seite 20 und folgende ausgeführt und weiter: «Die von den Tarifpartnern vereinbarten Massnahmen zur Steuerung der Kosten in gesamtschweizerischen Verträgen würden dann dazu dienen, die Zielvorgabe zu erreichen.» Später (Seite 45) werden in der Botschaft solche inhaltlichen Verbindungen dementiert: «Bei dieser Massnahme (Anmerkung: Steuerung der Kosten durch Tarifpartner) handelt es sich in kei- ner Weise um die Einführung eines Globalbudgets, da erstens keine Vorgabe eines Budgets vorgesehen ist und es zweitens weitgehend den Tarifpartnern überlassen wird, wie sie den Steuerungsmechanismus ausgestalten.» Über die Modalitäten der Steuerung gibt die Botschaft auf Seite 63 jedoch sehr wohl Hinweise. Die Steuerung des Leistungsbereichs sei in den Verträgen über die Vergütung, beispielsweise mit Tarifkürzungen, degressiven Tarifen oder Rückvergütungen, vorzusehen. Im Sozialversicherungsrecht wird ungenügende Tarifierung als Einschränkung einer versprochenen Leistung gewertet und damit als Verstoss gegen das Versicherungsprinzip. So wird im Bundesgerichtsurteil BGE 130 V 163 im Zusammenhang mit einem Versicherungsanspruch auf Hörgeräte festgehalten, dass das Bundesamt für Sozialversicherungen (BSV) fälschlicherweise von einem Vorrang des Tarifrechts gegenüber dem Leistungsrecht ausgegangen sei, welcher de lege lata nicht bestünde [2]. Keine Unterstützung für seine These erhält der Bundesrat zudem in der von ihm in Auftrag gegebenen Studie der B,S,S. Volkswirtschaftliche Beratung über die Globalbudgets in verschiedenen Kantonen. In Bezug auf die Wirkung seien die Instrumente Globalbudget, direkte Mengenbeschränkung und degressive Vergütung vergleichbar. Um die Wirkungen der Globalbudgets $\mathrm{zu}$ beurteilen, wird darin ein Vergleich zwischen den Kantonen, die Globalbudgets anwenden, und Kantonen, die dieses Instrument nicht nutzen, durchgeführt: «Neben Globalbudgets gemäss Art. 51 KVG können Kantone noch andere Instrumente der Mengensteuerung mit ähnlicher Wirkung anwenden (direkte Mengenbeschränkung, degressive Vergütung). Für die Analyse der Wirkung sind diese Kantone ebenfalls der Gruppe der Kantone mit Globalbudgets zuzuordnen» [3].

Das medizinisch Notwendige soll dem wirtschaftlich Möglichen geopfert werden. Dieses politische Anliegen, Leistungen in der Gesundheitsversorgung über ungenügende Tarifierung zu beschränken, soll also nach dem Willen des EDI an die Tarifpartner delegiert werden. Die Ärzteschaft steht jedoch für dessen Realisierung nicht zur Verfügung und wird diese für das hiesige Gesundheitswesen und die qualitativ hochstehende Versorgung der Patienten toxisch wirkende Tarifmassnahme bekämpfen.

Literatur

1 https://www.parlament.ch/de/ratsbetrieb/amtliches-bulletin/ amtliches-bulletin-die-verhandlungen?SubjectId $=43217$

2 http://relevancy.bger.ch/php/clir/http/index.php?highlight_ docid=atf\%3A\%2F\%2F130-V-163\%3Ait\&lang=it\&type=show_document

3 Seite xii B,S,S. Volkwirtschaftliche Beratung. Globalbudgets in den Kantonen im akutstationären Spitalbereich: Anwendung und Wirkungen unter der neuen Spitalfinanzierung. Im Auftrag des Bundesamts für Gesundheit. Mai 2019. 